\title{
CYSLTR1 promotes adenoid hypertrophy by activating ERK1/2
}

\author{
WENJIE GAO, JINYING LI, QUANHENG LI and SHUHUA AN \\ Department of Pulmonary Diseases, Children's Hospital of Hebei Province, Shijiazhuang, Hebei 050011, P.R. China
}

Received November 26, 2017; Accepted May 9, 2018

DOI: $10.3892 /$ etm.2018.6282

\begin{abstract}
Cysteinyl leukotriene receptor 1 (CYSLTR1) serves a pivotal role in allergic reactions, which is one of the main causes of adenoid hypertrophy. The present study aimed to investigate the function of CYSLT1 within adenoid hypertrophy. A total of 40 patients with adenoid hypertrophy were recruited between January 2014 and January 2016 at the Children's Hospital of Hebei Province, China. The patients were divided into either the mild-moderate group or the severe group according to their disease severity. The expression of CYSLT1 in the adenoid tissue and whole blood of all patients and healthy controls was detected by reverse transcription-quantitative polymerase chain reaction. Associations between the expression level of CYSLT1 and the clinical characteristics of patients were analyzed. Primary human adenoid epithelial cells (HAECs) with CYSLT1 knockdown and overexpression were constructed. The levels of extracellular signal-regulated kinase (ERK)2 and phosphorylated-ERK1/2 in adenoid tissue and HAECs were detected by western blot analysis. The expression of CYSLT1 in adenoid tissue and whole blood of all patients with adenoid hypertrophy was significantly higher compared with the healthy controls $(\mathrm{P}<0.05)$. In addition, the expression level of CYSLT1 was significantly higher in the severe group compared with the mild-moderate group $(\mathrm{P}<0.05)$. The highest level of p-ERK1/2 in adenoid tissue was observed in the severe group, followed by the mild-moderate group and then the control group $(\mathrm{P}<0.05)$. CYSLT1 expression was positively associated with the severity of disease. CYSLT1 knockdown significantly decreased the level of p-ERK1/2 in HAECs $(\mathrm{P}<0.05)$, while CYSLT1 overexpression significantly increased the level of p-ERK1/2. It was concluded that CYSLT1 may contribute to the progression of adenoid hypertrophy by activating ERK1/2.
\end{abstract}

Correspondence to: Dr Shuhua An, Department of Pulmonary Diseases, Children's Hospital of Hebei Province, 133 South Jianhua Street, Shijiazhuang, Hebei 050011, P.R. China

E-mail: qeuppu279@163.com

Key words: cysteinyl leukotriene receptor 1, adenoid hypertrophy, human adenoid epithelial cells, extracellular signal-regulated kinase $1 / 2$

\section{Introduction}

As one of the leading causes of nasal respiratory obstruction in childhood, adenoid hypertrophy affects $\sim 3 \%$ of children (1). The upper airway obstruction caused by adenoid hypertrophy may seriously affect maxillofacial and dental development (2). Besides that, nasal airway obstruction caused by this disease may lead to a series of problems including behavioral issues, night discomfort, daytime sleepiness and poor school performance (1). Moreover, adenoid hypertrophy is often combined with allergic rhinitis, which in turn increases the difficulties in the treatment and prolongs the duration of disease (3). Allergic diseases are closely correlated with the incidence of adenoid hypertrophy (4). Therefore, the inhibition of allergic reaction may benefit the prevention and treatment of adenoid hypertrophy.

As a receptor for cysteinyl leukotrienes, cysteinyl leukotriene receptor 1 (CYSLTR1) has been reported to play essential roles in mediating a variety of hypersensitivity and allergic reactions (5). It has been confirmed that the expression level of CYSLTR1 is abnormally upregulated in adenoid tissue of patients with adenoid hypertrophy, indicating the involvement of CYSLTR1 in the progression of this disease (6). However, the mechanism of the role of CYSLTR1 in adenoid hypertrophy remains unknown yet.

In the present study, expression of CYSLTR1 in adenoid tissue of patients with different degree of adenoid hypertrophy was detected. The correlation between the expression level of CYSLTR1 and clinical characteristics of patients were analyzed. In addition, the interaction between CYSLTR1 and extracellular signal-regulated kinase (ERK)1/2 was studied as well.

\section{Patients and methods}

Patients. A total of 40 patients with adenoid hypertrophy were selected during the period from January 2014 to January 2016 in Children's Hospital of Hebei Province (Shijiazhuang, China). All patients were diagnosed by symptoms including snoring, apnoea, nasal, congestion, and so on. Nasopharyngeal obstruction was detected using pharyngorhinoscopy or CT imagining. Patients with a history of allergy or allergy-related diseases, such as allergic rhinitis, were excluded from this study. There are 18 females and 22 males, and the age ranged from 4 to 8 years with an average age of $5.9 \pm 0.9$ years. The patients were divided into mild-moderate group $(<80 \%$ of the nasopharyngeal space had been occupied by the hypertrophied 
adenoid) and severe group ( $>80 \%$ of the nasopharyngeal space had been occupied by the hypertrophied adenoid). The mild-moderate group included 10 females and 14 males, and the age ranged from 4 to 8 years with an average age of 5.6 \pm 0.8 years. The severe group included 8 females and 8 males, and the age ranged from 4 to 8 years with an average age of $6.1 \pm 0.8$ years. At the same time, a total of 20 children without adenoid hypertrophy but needed surgical operations on adenoid due to other reasons were also included as control group. The control group included 10 females and 10 males, and the age ranged from 4 to 8 years with an average age of $6.9 \pm 1.1$ years. Tissue was collected from adenoid during surgical operations, and was immediately stored in liquid nitrogen before use. All participants' parents signed informed consent. The Ethics Committee of the Children's Hospital of Hebei Province approved this study.

Cell culture and transfection. Primary human adenoid epithelial cells (HAECs) were from the stock of our lab. This cell line was derived from a 6 years old child (male) who received adenoidectomy in our hospital. Cells were cultured with NHS medium supplemented with $10 \%$ heat inactivated horse serum (Biological Industries, Beit-Haemek, Israel) in an incubator $\left(37^{\circ} \mathrm{C}\right)$. Cells were cultured overnight to reach $80-90 \%$ confluent before transfection. CysLTR1 siRNA (h) (sc-43712) and control siRNA-A (sc-37007) were purchased from Santa Cruz Biotechnology (Dallas, TX, USA). The vector overexpressing CysLTR1 was constructed by inserting a EcoRI-EcoRI fragment containing the full length DNA sequence of CysLTR1 into pIRSE2-EGFP provided by Clontech (Palo Alto, CA, USA). Transfection was performed using Lipofectamine 2000 transfection reagent (11668-019) provided by Invitrogen (Thermo Fisher Scientific, Inc., Waltham, MA, USA). All operations were performed in strict accordance with the instructions provided by Invitrogen (Thermo Fisher Scientific, Inc.).

Real-time quantitative PCR. Total RNA was extracted from adenoid tissue using TRIzol reagent (Invitrogen; Thermo Fisher Scientific,Inc.). NanoDrop ${ }^{\mathrm{TM}} 2000$ Spectrophotometers (Thermo Fisher Scientific, Inc., Wilmington, DE, USA) was used to check the quality of all those RNA samples, and only the samples with A260/A280 ratio between 1.8 and 2.0 were used. SuperScript III Reverse Transcriptase (Thermo Fisher Scientific, Inc.) was used to carry out reverse transcription to synthesize cDNA. PCR reaction system was prepared using SYBR ${ }^{\circledR}$-Green Real-Time PCR Master Mixes provided by Thermo Fisher Scientific, Inc. The following primers were used: CysLTR1 forward, 5'-TGACCGCTGCCTTTT TAGTC-3' and reverse, 5'-AGGAGAGGGTCAAAGCAAC A-3'; $\beta$-actin forward, GACCTCTATGCCAACACAGT and reverse, AGTACTTGCGCTCAGGAGGA. PCR reaction conditions were: $95^{\circ} \mathrm{C}$ for $2 \mathrm{~min}$, followed by 40 cycles of $95^{\circ} \mathrm{C}$ for $20 \mathrm{sec}$ and $65^{\circ} \mathrm{C}$ for $40 \mathrm{sec}$. Expression level of CysLTR1 was normalized to endogenous control $\beta$-actin using $2^{-\Delta \Delta C T}$ method. PCR reactions were performed in triplicates, and each RNA sample was tested three times.

Western blot analysis. Peripheral blood $(5 \mathrm{ml})$ was extracted from each participant on the day of admission. Routine method was used to extract total protein from adenoid tissue, whole blood and HAECs. BCA method was used to test protein quality, followed by $10 \%$ SDS-PAGE gel electrophoresis to separate different proteins. Transmembrane was performed $(20 \mathrm{~V})$ to transfer protein to PVDF membrane. Membranes were blocked with 5\% skimmed milk, followed by incubation with corresponding primary antibodies including rabbit anti-p-ERK1/2 (Thr202/Tyr204) antibody (1:2,000; ab214362), anti-ERK2 antibody (1:2,000; ab32081) and anti-GAPDH (1:1,000; ab9845; all Abcam) overnight at $4^{\circ} \mathrm{C}$. Then membranes were washed with PBS, followed by incubating with IgG-HRP secondary antibody $(1: 1,000$; MBS435036; MyBioSource, San Diego, CA, USA) at room temperature for $2 \mathrm{~h}$. After washing with PBS, ECL method (Sigma-Aldrich; Merck KGaA, Darmstadt, Germany) was used to detect signals. Relative expression levels of ERK2 and p-ERK 1/2 were normalized to endogenous control GAPDH using Image $\mathbf{J}$ software.

Statistical analysis. All statistical analyses were performed using SPSS 19.0 (SPSS Inc., USA). Comparison of normal distribution data $(\overline{\mathrm{x}} \pm \mathrm{s})$ between two group were performed by $\mathrm{t}$ test, and comparisons among multiple groups were performed by one-way analysis of variance and LSD test. Comparisons of non-normal distribution data were performed by non-parametric Mann-Whitney $\mathrm{U}$ test. $\mathrm{P}<0.05$ was considered to indicate a statistically significant difference.

\section{Results}

Expression of CYSLTR1 in adenoid tissue and whole blood of patients with adenoid hypertrophy. Expression of CYSLTR1 in adenoid tissue and whole blood of patients with adenoid hypertrophy were detected by qRT-PCR. As shown in Fig. 1A, compared with control group, expression level of CYSLTR1 in adenoid tissue of patients in mild-moderate group and severe group were significant increased $(\mathrm{P}<0.05)$. Besides that, compared with mild-moderate group, expression level of CYSLTR1 in adenoid tissue was also significantly increased in patients of severe group $(\mathrm{P}<0.05)$. Similar results were found in the expression of CYSLTR1 in whole blood of patients in different groups. As shown in Fig. 1B, highest expression level of CYSLTR1 was found in severe group, followed by mild-moderate group and control group $(\mathrm{P}<0.05)$. Those results suggest that CYSLTR1 is involved in the development of adenoid hypertrophy, and the expression level of CYSLTR1 is positively correlated with the severity of this disease.

Correlation between the expression levels of CYSLTRI and the pathological stages. Expression levels of CYSLTR1 in both adenoid tissue and whole blood were divided into high expression group and low expression group according to the median value of relative expression level. Correlations between the expression levels of CYSLTR1 in both adenoid tissue and whole blood and the clinical data of patients including age, sex, and pathological stages were analyzed. As shown in Tables I and II, expression levels of CYSLTR1 in adenoid tissue and whole blood showed no significant correlations with age and sex, but showed significant correlations with the 

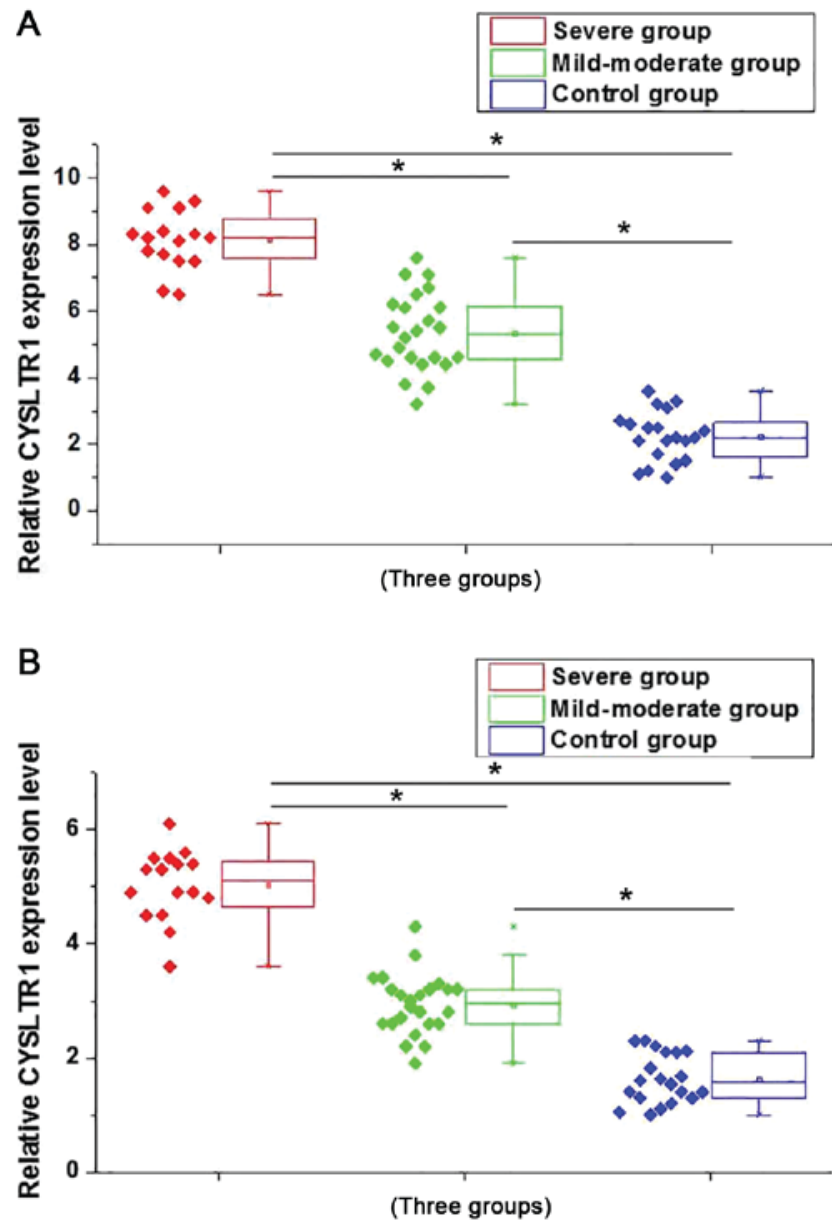

Figure 1. Expression of CYSLTR1 in adenoid tissue and whole blood of patients in different groups. (A) Expression of CYSLTR1 in adenoid tissue of patients in different groups. (B) Expression of CYSLTR1 in whole blood of patients in different groups. "P<0.05. CYSLTR1, cysteinyl leukotriene receptor 1 .

severity of disease, and higher degree of severity was followed by the higher expression level of CYSLTR1.

Effects of adenoid hypertrophy on the activation of ERK1/2. The activation of ERK1/2 is involved in various allergic reactions, which are the main causes of adenoid hypertrophy. Therefore, the phosphorylation of in ERK1/2 in adenoid tissue of three randomly selected participants was detected by western blot. As shown in Fig. 2, no significant differences in expression levels of ERK2 were found between different groups of patients. However, levels of p-ERK1/2 were significantly higher in patients of severe group and mild-moderate group than in control group $(\mathrm{P}<0.05)$. In addition, compared with mild-moderate group, levels of p-ERK1/2 were also significantly increased in patients of severe group $(\mathrm{P}<0.05)$. Those data suggest that the activation of ERK $1 / 2$ is involved in the development of adenoid hypertrophy and higher activation levels indicate higher degree of severity.

Effects of CYSLTR1 silencing and overexpression on phosphorylation of ERK1/2 in HAECs. It is known that CYSLTR1 can interact with ERK1/2 to perform its biological functions.
In this study, CYSLTR1 was silenced and overexpressed in HAECs. CYSLTR1 silencing was confirmed by measuring the expression level of CYSLTR1by qRT-PCR (data not show) to investigate the interactions between CYSLTR1 and ERK1/2. As shown in Fig. 3, no significant differences in expression levels of ERK2 were found between different groups of cells. Compared with control group, level of p-ERK1/2 was significant reduced in HAECs with CYSLTR1 silencing and significant increased in HAECs with CYSLTR1 overexpression $(\mathrm{P}<0.05)$. Those data suggest that CYSLTR1 can regulate the activation of ERK1/2 to participate in the development of adenoid hypertrophy.

\section{Discussion}

Allergic reactions were found to be highly associated with the development of adenoid hypertrophy in both children and adults, and $\sim 60 \%$ of the patients show different degrees of allergic manifestations (7). In another study, Huang and Giannoni reported that sensitivity to mold allergens was a major risk factor for adenoid hypertrophy in children with allergic rhinitis, which prevents children from the exposure to molds bringing beneficial effects to the prevention of adenoid hypertrophy (8). Cysteinyl leukotriene receptors including CYSLTR1 play pivotal roles in mediating airway remodeling caused by allergic reactions by binding to those cysteinyl leukotrienes (9). The polymorphism of CYSLTR1 was also found to be correlated with the hypersensitivity to foods and drugs, such as aspirin (10). The involvement of CYSLTR1 in adenoid hypertrophy still has not been well studied except one recent study reporting that expression level of CYSLTR1 was significantly increased in adenoid tissues of children with adenoid hypertrophy compared with the healthy children (6). Concordant results were found in this study. In this study, expression level of CYSLTR1 was found to be significantly higher in the adenoid tissue and whole blood of children with adenoid hypertrophy than in healthy control children. In addition, expression level of CYSLTR1 was also higher in patients with severe adenoid hypertrophy than in patients with mild-moderate adenoid hypertrophy. There are possibly two different resources of CYSLTR1 in blood. It is known that blood leukocytes can express CYSLTR1 (11). Circulating CYSLTR1 may also exist in blood. However, this uncertainty may not affect our conclusions. Besides that, expression level of CYSLTR1 in adenoid tissue and whole blood of children with adenoid hypertrophy showed no significant correlations with age and sex, but it was significantly correlated with the severity of disease. Those results suggest that CYSLTR1 is involved in the development of adenoid hypertrophy and expression level of CYSLTR1 is positively correlated with the severity of the disease.

As two related protein-serine/threonine kinases, ERK1 and ERK2, or ERK1/2 are two key players in Ras-Raf-MEK-ERK signal transduction cascade, which has been confirmed to be with pivotal roles in the regulation of various biological processes including cell survival, migration, adhesion, proliferation, differentiation and metabolism, and cell cycle progression (12). Recent studies have also shown that the activation of ERK1/2 also has important 
Table I. Correlation between the expression level of CYSLTR1 in adenoid tissue and the clinical characteristics of patients.

\begin{tabular}{|c|c|c|c|c|c|}
\hline \multirow[b]{2}{*}{ Clinicopathological features } & \multirow[b]{2}{*}{ Groups } & \multirow[b]{2}{*}{ Total } & \multicolumn{2}{|c|}{ CYSLT1 expression } & \multirow[b]{2}{*}{ P-value } \\
\hline & & & High & Low & \\
\hline \multirow[t]{2}{*}{ Sex } & Female & 18 & 9 & 9 & \multirow[t]{2}{*}{0.77} \\
\hline & Male & 22 & 10 & 12 & \\
\hline \multirow[t]{2}{*}{ Age (years) } & $>6$ & 22 & 11 & 11 & \multirow[t]{2}{*}{0.48} \\
\hline & $\leq 6$ & 18 & 7 & 11 & \\
\hline \multirow[t]{2}{*}{ Stage } & Severe & 16 & 13 & 3 & \multirow[t]{2}{*}{$<0.00001$} \\
\hline & Mild-moderate & 24 & 2 & 22 & \\
\hline
\end{tabular}

CYSLTR1, cysteinyl leukotriene receptor 1 .

Table II. Correlation between the expression level of CYSLTR1 in whole blood and the clinical characteristics of patients.

\begin{tabular}{|c|c|c|c|c|c|}
\hline \multirow[b]{2}{*}{ Clinicopathological features } & \multirow[b]{2}{*}{ Groups } & \multirow[b]{2}{*}{ Total } & \multicolumn{2}{|c|}{ CYSLT1 expression } & \multirow[b]{2}{*}{ P-value } \\
\hline & & & High & Low & \\
\hline \multirow[t]{2}{*}{ Sex } & Female & 18 & 11 & 7 & \multirow[t]{2}{*}{0.90} \\
\hline & Male & 22 & 13 & 9 & \\
\hline \multirow[t]{2}{*}{ Age (years) } & $>6$ & 22 & 12 & 10 & \multirow[t]{2}{*}{0.53} \\
\hline & $\leq 6$ & 18 & 8 & 10 & \\
\hline \multirow[t]{2}{*}{ Stage } & Severe & 16 & 15 & 1 & \multirow[t]{2}{*}{$<0.00001$} \\
\hline & Mild-moderate & 24 & 2 & 22 & \\
\hline
\end{tabular}

CYSLTR1, cysteinyl leukotriene receptor 1 .

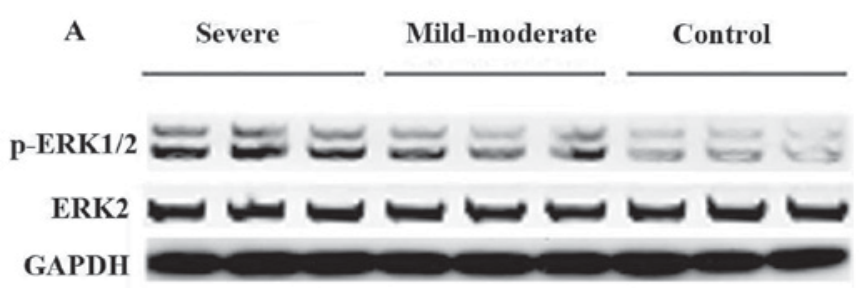

B

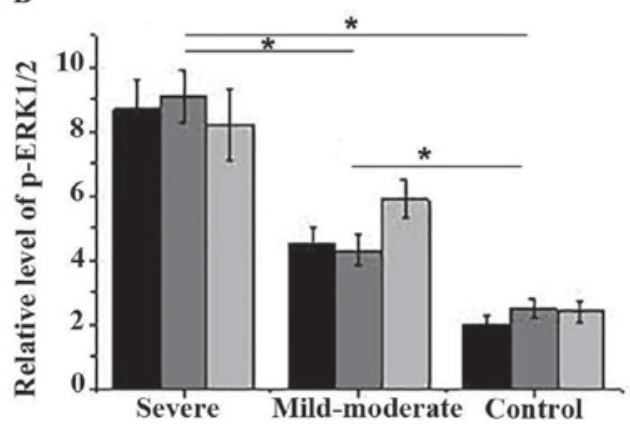

Figure 2. Effects of adenoid hypertrophy on the activation of ERK1/2 (A) Representative results of western blot analysis. (B) Normalized level of $\mathrm{p}$-ERK1/2 in each group. ${ }^{*} \mathrm{P}<0.05$. ERK, extracellular signal-regulated kinase; p, phosphorylated.

functions in allergic responses and the inhibition of ERK1/2 activation can help reduce allergic inflammation (13). In the

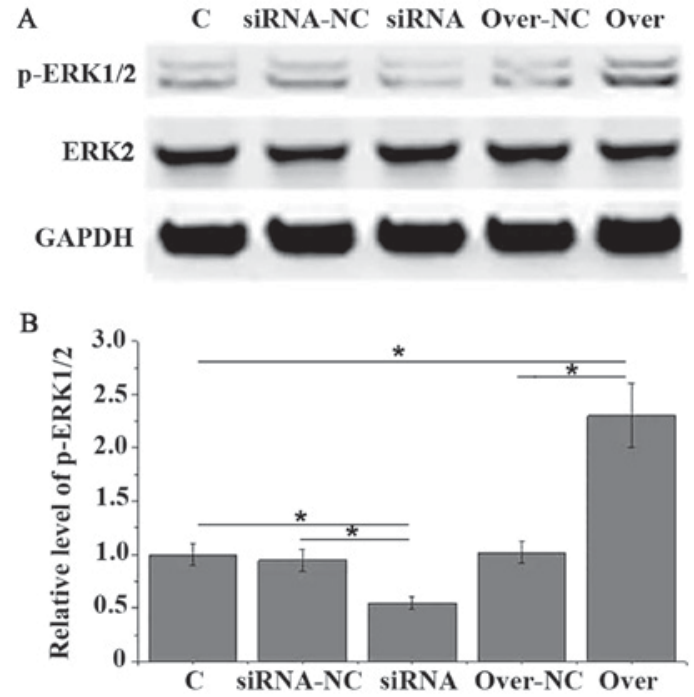

Figure 3. Effects of CYSLTR1 silencing and overexpression on phosphorylation of ERK1/2 in human adenoid epithelial cells. (A) Representative results of western blot analysis. (B) Normalized level of p-ERK1/2 in different groups of cells. ${ }^{*} \mathrm{P}<0.05$. ERK, extracellular signal-regulated kinase; CYSLTR1, cysteinyl leukotriene receptor 1; p, phosphorylated; si, small interfering; NC, negative control; over-NC, transfection with empty pIRSE2-EGFP.

study of allergen-induced lung inflammation, Chen et al reported that downregulation of ERK1/2 could inhibit the 
development of bronchial asthma by reducing inflammatory responses (14). In another study, ERK1/2 kinase inhibition was found to be able to attenuate Th2 immune response induced by allergens in mouse model (15). Based on our knowledge, the involvement of ERK1/2 kinase in the development of adenoid hypertrophy still has not yet been reported. In our study, no significant differences in expression of ERK2 were found between different groups. However, the level of p-ERK1/2 was significantly higher in patients with adenoid hypertrophy than in healthy children. In addition, the level of p-ERK1/2 was also significantly higher in severe group than in mild-moderate group. Those data suggest that the activation of $\mathrm{p}$-ERK1/2 is involved in the progression of adenoid hypertrophy.

It has been reported that the biological functions of CYSLTR1 in allergic responses is closely related to the activation of ERK $1 / 2$ signaling pathway (16). In the present study, HAECs with CYSLTR1 overexpression and knockdown were constructed to investigate the interactions between CYSLTR1 and ERK1/2. No significant differences in the expression level of ERK2 were found among different groups of cells, but the level of p-ERK1/2 was found to be significantly higher in HAECs with CYSLTR1 overexpression compared with control cells. In contrast, level of p-ERK1/2 was significantly decreased in HAECs with CYSLTR1 knockdown. Those results suggest that CYSLTR1 has no effects on the expression level of ERK2, but CYSLTR1 expression can induce the activation of ERK1/2 through phosphorylation, so as to increase allergic reaction and promote the development of adenoid hypertrophy.

In conclusion, expression level of CYSLTR1 is positively correlated with the progression of adenoid hypertrophy. CYSLTR1 expression can induce the activation of ERK1/2 to contribute to the development of adenoid hypertrophy. This study provided the basis for future studies, which aim to investigate the clinical value of CYSLTR 1 in the treatment of adenoid hypertrophy. Although this study has the unavoidable limitation of small sample size, the results are representative and convincing. Future studies with bigger sample size will further confirm the conclusions that are made in the present study.

\section{Acknowledgements}

Not applicable.

\section{Funding}

No funding was received.

\section{Availability of data and materials}

All data generated or analyzed during this study are included in this published article.

\section{Authors' contributions}

WG and SA designed experiments; WG and JL performed experiments, WG, JL and QL analyzed data; SA wrote the manuscript; all authors read the manuscript.

\section{Ethics approval and consent to participate}

The Ethics Committee of the Children's Hospital of Hebei Province approved this study. All participants' parents signed informed consent for their inclusion within the study.

\section{Consent for publication}

All participants' parents signed informed consent for the publication of their child's data.

\section{Competing interests}

The authors declare that they have no competing interests.

\section{References}

1. Koca CF, Erdem T and Bayındır T: The effect of adenoid hypertrophy on maxillofacial development: An objective photographic analysis. J Otolaryngol Head Neck Surg 45: 48, 2016.

2. Raffat A and ul Hamid W: Cephalometric assessment of patients with adenoidal faces. J Pak Med Assoc 59: 747-752, 2009.

3. Liu W, Zhou L, Zeng Q and Luo R: Combination of mometasone furoate and oxymetazoline for the treatment of adenoid hypertrophy concomitant with allergic rhinitis: A randomized controlled trial. Sci Rep 7: 40425, 2017.

4. Evcimik MF, Dogru M, Cirik AA and Nepesov MI: Adenoid hypertrophy in children with allergic disease and influential factors. Int J Pediatr Otorhinolaryngol 79: 694-697, 2015.

5. Honda T and Kabashima K: Leukotrienes as key mediators and amplifiers in allergic inflammation: Insights from the bench and clinic. Exp Dermatol 23: 95-96, 2014.

6. Zhu MH, Liang M, Wang ZJ, Shen Y, Wen HY, Zhai JM and Yang XB: Expression of CysLTR-1 and CysLTR-2 in adenoid tissues from children with adenoid hypertrophy. Zhongguo Dang Dai Er Ke Za Zhi 17: 159-163, 2015 (In Chinese).

7. Al-juboori AN, Fadhil A and Sahib N: Adenoid hypertrophy in adult patients and its relation with allergy. Eur Sci J 10: 481-487, 2014.

8. Huang SW and Giannoni C: The risk of adenoid hypertrophy in children with allergic rhinitis. Ann Allergy Asthma Immunol 87: 350-355, 2001.

9. Henderson WR Jr, Chiang GK, Tien YT and Chi EY: Reversal of allergen-induced airway remodeling by CysLT1 receptor blockade. Am J Respir Crit Care Med 173: 718-728, 2006.

10. Kim SH, Ye YM, Hur GY, Lee SK, Sampson AP, Lee HY and Park HS: CysLTR1 promoter polymorphism and requirement for leukotriene receptor antagonist in aspirin-intolerant asthma patients. Pharmacogenomics 8: 1143-1150, 2007.

11. Roskoski R Jr: ERK1/2 MAP kinases: Structure, function, and regulation. Pharmacol Res 66: 105-143, 2012.

12. Figueroa DJ, Breyer RM, Defoe SK, Kargman S, Daugherty BL, Waldburger K, Liu Q, Clements M, Zeng Z, O'Neill GP, et al: Expression of the cysteinyl leukotriene 1 receptor in normal human lung and peripheral blood leukocytes. Am J Respir Crit Care Med 163: 226-233, 2001.

13. El-Hashim AZ, Renno WM, Raghupathy R, Abduo HT, Akhtar S and Benter IF: Angiotensin-(1-7) inhibits allergic inflammation, via the MAS1 receptor, through suppression of ERK1/2- and NF- $\kappa$ B-dependent pathways. Br J Pharmacol 166: 1964-1976, 2012.

14. Chen H, Xu X, Teng J, Cheng S, Bunjhoo H, Cao Y, Liu J, Xie J, Wang C, Xu Y and Xiong W: CXCR4 inhibitor attenuates allergen-induced lung inflammation by down-regulating MMP-9 and ERK1/2. Int J Clin Exp Pathol 8: 6700-6707, 2015.

15. Saw S and Arora N: PI $3 K$ and ERK1/2 kinase inhibition potentiate protease inhibitor to attenuate allergen induced Th2 immune response in mouse. Eur J Pharmacol 776: 176-184, 2016.

16. Paulucci BP, Pereira J, Picciarelli P, Levy D and Di Francesco RC: Expression of cysteinyl leukotriene receptor 1 and 2 (CysLTR1 and CysLTR2) in the lymphocytes of hyperplastic tonsils: Comparison between allergic and nonallergic snoring children. Int Forum Allergy Rhinol 6: 1151-1158, 2016. 